\title{
Redução do quadro álgico em pacientes com fibromialgia através do exercício físico resistido, orientado e acompanhado pelo profissional de educação física
}

\author{
Reduction of pain in patients with fibromyalgia through physical exercise \\ registered, guided and accompanied by a physical education professional
}

Thiago Bruno Souto de Oliveira ${ }^{1 *}$, Neide Kazue Sakugawa Shinohara ${ }^{2}$

\begin{abstract}
RESUMO
O objetivo é apresentar uma revisão bibliográfica sobre a melhora global que o exercício físico resistido, acompanhado pelo profissional de educação física promove na redução do quadro álgico em mulheres diagnosticadas com fibromialgia. O levantamento bibliográfico foi realizado de 1990 a 2021, nas plataformas Medline, Lilacs, SciELO, a partir dos descritores "musculação", "exercícios físico", em combinação com "fibromialgia" e "mulheres", nos vernáculos em inglês, espanhol e português. Foram identificados estudos clínicos/atividade física que abordaram os efeitos causados pela prática do exercício físico em pacientes diagnosticadas com fibromialgia, analisando se produzia efeitos benéfico ou maléfico. No levantamento foi constatado que a prática de exercício físico, moderada e supervisionada por profissionais, possibilitou a diminuição de quadros álgico em mulheres com síndrome de fibromialgia. As evidências cientificas indicam que a prática do exercício físico resistido, causa um efeito analgésico por estimular a liberação de endorfinas; funcionar como antidepressivo; e proporcionar uma sensação de bemestar global e de autocontrole para atividades independentes.
\end{abstract}

Palavras-chave: Musculação; Exercícios Físico; Fibromialgia; Mulheres;

\begin{abstract}
The objective is to present a literature review on the global improvement that resistance physical exercise, accompanied by a physical education professional, promotes in the reduction of pain in women diagnosed with fibromyalgia. The bibliographic survey was carried out from 1990 to 2021, on Medline, Lilacs, SciELO platforms, using the descriptors "weight lifting", " exercises physical", in combination with "fibromyalgia" and "women", in the vernaculars in English, Spanish and Portuguese. Clinical/physical activity studies that addressed the effects caused by the practice of physical exercise in patients diagnosed with fibromyalgia were identified, analyzing whether it produced beneficial or harmful effects. In the survey, it was found that the practice of physical exercise, moderated and supervised by professionals, enabled the reduction of pain in women with fibromyalgia syndrome. Scientific ev idence indicates that
\end{abstract}

\footnotetext{
${ }^{1}$ Bacharelando em Educação Física, Centro Universítário Brasileiro - UNIBRA

*E-mail: thiagobruno211@gmail.com

${ }^{2}$ Docente da Universidade Federal Rural de Pernambuco - UFRPE
} 
the practice of resistance physical exercise causes an analgesic effect by stimulating the release of endorphins; function as an antidepressant; and provide a sense of overall well-being and self-control for independent activities.

Keywords: Bodybuilding; Exercises Physical; Fibromyalgia; Women;

\section{INTRODUÇÃO}

Segundo o American College of Rheumatology - ACR (2019), a Fibromialgia (FM) é uma condição neurológica crônica que causa dor em diferentes regiões do corpo: dor axial, dor nos lados esquerdo e direito e nos segmentos superior e inferior, dentre outros sintomas associados. A dor e a sensibilidade provocada pela fibromialgia tendem a ir e vir, e essas sensações tornam-se bastante incômodas. Na maioria das vezes, as pessoas com essa doença ficam muito cansadas, irritadas, estressadas o que impacta diretamente na qualidade do sono. Embora a FM possa afetar significativamente a qualidade de vida nos humanos devido a limitação imposta pela dor generalizada, em princípio não incorre em relação direta com casos de ataques cardíacos, derrames, cânceres, ou morte por stress físico.

Normalmente existem alguns gatilhos que evoluem para um desenvolvimento progressivo de quadros, como: problemas de coluna, artrite, lesões, estresse físico e de componente emocional. O resultado é uma mudança na maneira como o corpo "se comunica" com a medula espinhal e o cérebro. A fibromialgia não é um distúrbio autoimune, uma inflamação ou um problema muscular ou articular. Provavelmente, existem certos genes que tornam as pessoas mais propensas à $\mathrm{FM}$ e outros problemas de saúde associados a essa patologia. No entanto, a influência genética por si só não é a causa para evolução de FM e seu quadro evolutivo de cronificação. A clínica médica leva também em consideração outros fatores fisiológicos, ambiental e comportamental. O diagnóstico pode ser feito com um exame clínico cuidadoso, pois não há nenhum teste bioquímico específico para detectar esta doença crônica. Frequentemente tem sido um diagnóstico baseado em sinais e sintomas, complementado por exames para detecção de marcadores de infecção e raios-X dos membros afetados (ACR, 2019).

Borchers e Gershwin (2015) relatam que em 1990 a Associação Americana - ACR definiu os critérios diagnósticos para a presença de FM, como dor generalizada no lado 
esquerdo e direito, sensibilidade à dor em pelo menos 11 dos 18 locais nas regiões do corpo especificados como indicativo de FM, associado a relatos dos pacientes como uma dor crônica e persistente, afetando de dois a quatro por cento das pessoas no mundo, a questão de gênero se mostra importante, as mulheres aparecem com mais frequência nos diagnósticos dessa patologia, quando comparados com os homens, demonstrando o impacto social na força feminina na esfera profissional e doméstica.

Souza e Perissinotti (2018), relatam que no Brasil a prevalência da Síndrome de Fibromialgia (SFM) está estimada em $2 \%$ da população brasileira, estabelecendo uma relação por gênero, essa doença afeta na proporção de 1 homem para 5,6 mulheres. Nessa pesquisa os achados confirmam que a SFM é mais prevalente em mulheres da raça branca na faixa etária de 35 a 55 anos.

Não há cura e tratamento eficaz para reverter o quadro da FM, os sintomas podem ser amenizados com tratamentos não medicamentosos como exercícios de fisioterapia, exercício físico resistido, sendo estes de baixo impacto, contínuo e acompanhado, terapias complementares com massagem muscular, pilates, além do uso de medicamentos relaxantes, antidepressivos e neuromoduladores. Publicações que tratam da fisiologia do esporte e exercício mostram que o tratamento mais eficaz e recomendado para amenizar o quadro álgico da SFM é o exercício físico resistido moderado, acompanhado pelo profissional de educação física (ACR, 2019; WOLFE et al., 2010).

Corrales e Lora (2007), devido à dor generalizada e muitas vezes limitantes para realização de atividades simples e rotineiras, as pessoas com fibromialgia (FM), têm dificuldade em aderir a programas de exercícios físicos, mesmo quando são recomendados clinicamente para mitigar a progressão da doença. Levando essas restrições individuais em consideração, o objetivo desse estudo foi verificar a eficácia de como um programa de treinamento de flexibilidade e de força acompanhado por um profissional de educação física, se constitui em poderosa ferramenta de conduta clínica, que pode amenizar as dores incapacitantes em pacientes com FM.

\section{Quadro clínico da síndrome de fibromialgia}

Segundo o Ministério da Saúde que utiliza a classificação do Código Internacional de Doenças (CID), publicação elaborada e editada pela Organização Mundial de Saúde 
(OMS), visando padronizar a codificação de doenças e outros problemas relacionados à saúde, como instrumento de consulta de base epidemiológica, organizando informações científicas, sinais, sintomas, achados anormais, queixas, circunstâncias sociais e causas externas, no estudo de doenças que afetam determinado local ou grupo de pessoas, para que seja realizada ações de interesse público da forma mais correta, simples e universal. Segundo o CID, para o portador de Fibromialgia (FM), deverá constar com o CID 10 M79.7, descrição que identifica internacionalmente que o paciente é portador dessa patologia crônica, o que possibilita mover ações trabalhista por incapacidade física para o trabalho (TANGERINO, 2014).

Haun et al. (1999), descrevem em seu artigo que a síndrome da fibromialgia pode ser definida como uma síndrome dolorosa crônica, de etiologia desconhecida, que se manifesta no sistema músculo esquelético. Com isso, na tentativa de estudar a epidemiologia da população acometida de FM, o American College of Rheumatology (1990), publicou critérios de classificação da fibromialgia. Dentre os protocolos de diagnóstico, destacam-se uma sensibilidade dolorosa em sítios anatômicos preestabelecidos, denominados “tender points" (pontos sensíveis) ao toque. O número de tender points relaciona-se com avaliação global da gravidade das manifestações clínicas, fadiga, distúrbio do sono, depressão, ansiedade e não raro o relato de vários sintomas associados simultaneamente.

Faz-se importante ressaltar que estes "tender points" (Figura 1), não são geralmente conhecidos pelos pacientes e normalmente não se situam na zona central da dor por eles referida na clina médica. De acordo com os critérios atuais, devem ser pesquisados os seguintes de pontos:

1. Sub-occipital - na inserção do músculo sub-occipital;

2. Cervical baixo - atrás do terço inferior do esternocleidomastóideo, no ligamento inter-transverso C5-C6;

3. Trapézio - ponto médio do bordo superior, numa parte firme do músculo;

4. Supra- espinhoso - acima da escápula, próximo à borda mediaI, na origem do músculo supra-espinhoso;

5. Segunda junção costocondral - lateral à junção, na origem do músculo grande peitoral;

6. Epicôndilo lateral - 2 a 5 em de distância do epicôndilo lateral; 
7. Glúteo médio - na parte média do quadrante súpero externo na porção anterior do músculo glúteo médio;

8. Trocantérico - posterior à proeminência do grande trocanter;

9. Joelho - no coxim gorduroso, pouco acima da linha média do joelho.

Figura 1- Os 18 Pontos dolorosos (tender points).

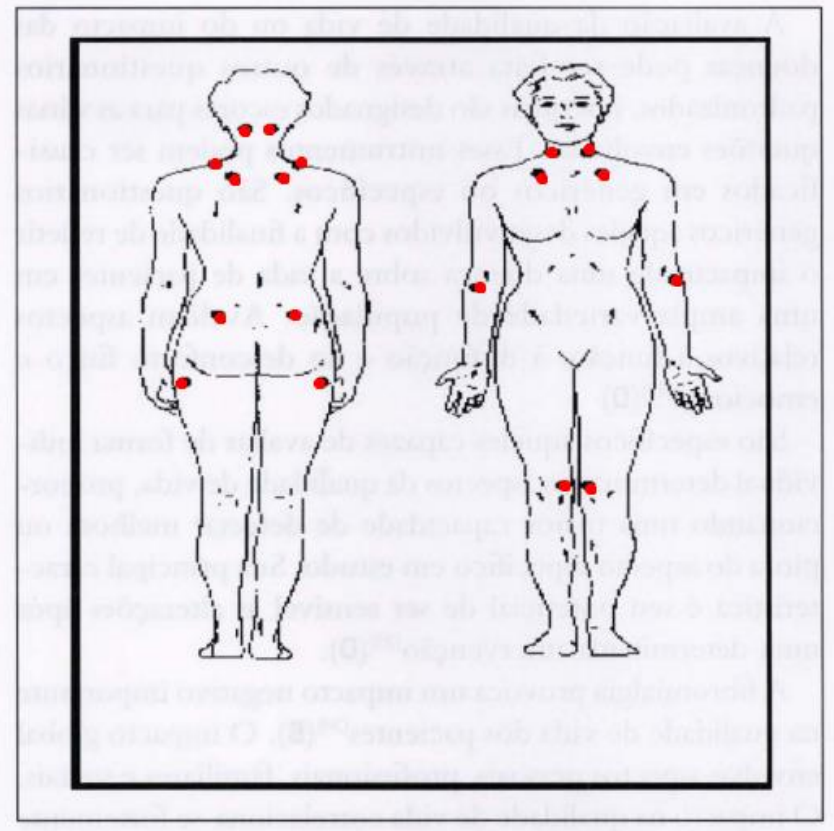

Fonte: American College of Rheumatology. (1990)

Russell (1992), aborda que o quadro clínico desta síndrome costuma ser polimorfo, exigindo anamnese cuidadosa e exame físico detalhado. $\mathrm{O}$ sintoma presente em todos os pacientes é a dor muscular difusa e crônica, envolvendo o esqueleto axial e periférico. Em geral, os pacientes têm dificuldade para localizar a dor, muitas vezes apontando sítios periarticulares (que se encontra ao redor de uma articulação, ponto de junção de ossos ou partes ósseas), sem especificar o ponto exato da dor.

Embasado em Provenza et al. (2004), que descrevem a não comprovação da hipótese de que a fibromialgia possa ser uma variante da doença depressiva, porque já está consolidado o uso de protocolos clínicos caracterizando a fibromialgia como sendo um problema puramente psicológico ou puramente orgânico. Os estudos científicos apontam que a SFM ativa uma disfunção no processo sensorial, induzindo que a dor 
desses pacientes diagnosticados é real, e que outros sintomas que venha a amostra podem ser secundários a dor.

Em buscas esclarecedoras sobre os sintomas da SFM, um dos achados foi da Bilodeau (2020), afirmando que pessoas com fibromialgia apresentam dores generalizadas, difusas, rigidez nos músculos e articulações, bem como cansaço incomum. Uma das principais teorias é que isso se deve a um funcionamento anormal do cérebro que amplifica as respostas nervosas normais, fazendo com que as pessoas com fibromialgia experimentem dor em vários locais do corpo, gerando um quadro de desconforto crônico e generalizado.

Provenza e colaboradores (2004), denotam que geralmente o sintoma mais presente é a "sensação" de inchaço, particularmente nas mãos, antebraços e trapézios e não está relacionada a qualquer processo inflamatório. Além dessas manifestações músculo-esqueléticas, muitos se queixam de sintomas não relacionados ao aparelho locomotor. Entre esta variedade de queixas, destaca-se cefaleia, tontura, zumbido, dor torácica atípica, palpitação, dor abdominal, constipação, diarreia, dispepsia, tensão prémenstrual, urgência miccional, dificuldade de concentração e falta de memória. No estudo de Junior e colaboradores (2012), abordam os seguintes sintomas: A ausência de sono pelo fato da SFM intencionar um quadro elevado de dores. A fadiga, sendo bastante significativa com sensação de exaustão fácil, levando a dificuldades para realização de tarefas laborais ou domésticas.

Resultados do treinamento de força resistido e flexibilidade em pacientes com fibromialgia, acompanhado pelo profissional de educação física

Oliveira et al. (2017), relatam em seu estudo o efeito do exercício físico, supervisionado durante 6 meses, sobre a flexibilidade mobiliar em 29 mulheres, com idade de 48,6 $\pm 10,3$ anos, diagnosticada com a síndrome da fibromialgia (SFM). O treinamento de força e flexibilidade, foram compostos por 10-11 exercícios, monoarticular e multiarticular, enquadrados nos tender points mostrado pelo American College of Rheumatology (1990). Contribuindo para uma execução de movimentos eficientes e manutenção do equilíbrio, os autores realizaram duas sessões de exercício físico supervisionado por semana, durando uma hora em 6 meses, envolvendo o 
treinamento de força, da flexibilidade e resistência aeróbica (TABELA 1). Sendo que a flexibilidade, assim como a força muscular e a resistência aeróbica, é uma valência física necessária para a consecução das atividades da vida diária e conservação da saúde. Objetivando o desenvolvimento da aptidão física relacionada a saúde, os autores salientam que essas pacientes também tiveram orientação nutricional quinzenal e apoio psicológico semanal por profissionais habilitados. Mostrando um resultado significativamente positivo na flexibilidade após seis meses de exercícios físicos supervisionado com o apoio nutricional e psicológico.

O estudo de Bulhões et al. (2018), promoveu um levantamento que abordava o efeito do treinamento de força resistido e acompanhado na redução da dor em mulheres com idade média de 48 anos, diagnosticadas com FM. Os autores abordaram que o Colégio Americano de Medicina Esportiva, afirma que o exercício resistido, realizado 23 vezes por semana, com série de 8-12 repetições, com duração de pelo menos 30 min, são muito eficazes na redução da dor em pacientes com FM. Vale salientar que toda a carga foi incrementada de acordo com a tolerância do paciente, respeitando suas individualidades. Foram constatados nas análises dos estudos levantados por Bulhões e colaboradores (TABELA 1), uma redução do quadro álgico de forma estatisticamente significativa nas pacientes diagnosticada com SFM.

Tabela 1- Respostas significativas do treinamento de força e flexibilidade na redução do quadro álgico em mulheres com fibromialgia

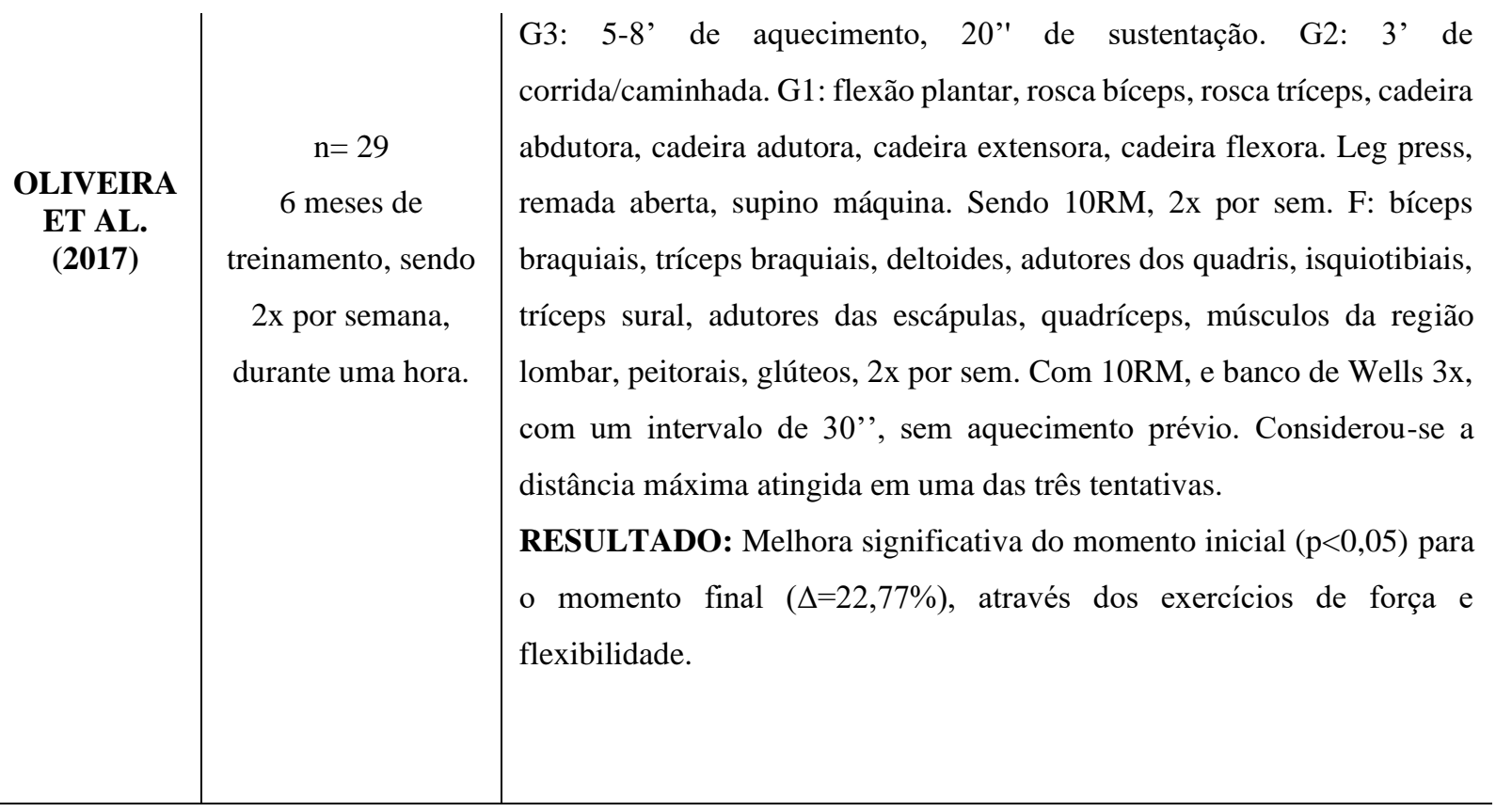




\begin{tabular}{|c|c|c|}
\hline $\begin{array}{c}\text { BIRCAN } \\
\text { ET AL. } \\
\text { (2008) }\end{array}$ & $\begin{array}{c}\mathrm{n}=26 \\
\text { G1: } \mathrm{n}=13 \\
\text { G2: } \mathrm{n}=13\end{array}$ & $\begin{array}{l}\text { ER em pé, sentado e deitado. Iniciando com 4-5 rep. progredindo para } 12 \text {, } \\
\text { com uso de pesos livres e peso do corpo. 5' de aquecimento, 30' de ER e } \\
\text { 5' de desaquecimento. G2: Caminhada de } 20-30 \text { ', ajustada em } 60-70 \% \text { da } \\
\text { frequência cardíaca. Todos: } 3 x / S e m \text {, por } 8 \text { sem. } \\
\text { RESULTADO: Diminuição da dor }(p<0,05) \text { em ambos os grupos. }\end{array}$ \\
\hline $\begin{array}{c}\text { GAVI ET } \\
\text { AL. } \\
(2014)\end{array}$ & $\begin{aligned} \mathrm{n} & =66 \\
\text { G1: } & =35 \\
\text { G3: } \mathrm{n} & =31\end{aligned}$ & $\begin{array}{l}\text { G1: Exercício com sobrecarga de } 45 \% \text { de } 1 \mathrm{RM} \text { para: quadríceps, } \\
\text { isquiotibiais, bíceps e tríceps branquial, peitoral, tríceps sural, deltoide e } \\
\text { latíssimo do dorso, em } 12 \text { exercícios com } 3 \text { séries de } 12 \text { rep., com duração } \\
\text { de } 45^{\prime} \text {. G3: Incluiu alongamento da maioria dos músculos. Todos: } \\
\text { 2x/sem, por } 16 \text { sem. } \\
\text { RESULTADO: Melhora da dor em ambos os grupos. Porém, o efeito no } \\
\text { grupo G1 foi maior do que o grupo G3, após o } 1^{\circ} \text { e o } 4^{\circ} \text { mês }(p<0,05) \text {. }\end{array}$ \\
\hline $\begin{array}{c}\text { KAYO ET } \\
\text { AL. } \\
(\mathbf{2 0 1 2})\end{array}$ & $\begin{array}{c}\mathrm{n}=90 \\
\mathrm{G} 1: \mathrm{n}=30 \\
\mathrm{G} 2: \mathrm{n}=30 \\
\mathrm{G} 4: \mathrm{n}=30\end{array}$ & $\begin{array}{l}\text { G1: } 11 \text { exercícios ativos livres, com pesos livres e peso do corpo, na } \\
\text { posição sentado, deitado e em pé. Realizaram } 3 \text { séries de } 10-15 \text { rep. com } \\
\text { repouso de 1'. G2: Caminhada inicialmente por } 25-30^{\prime} \text {, progredindo para } \\
\text { 50', bem como a intensidade do exercício. G4: Avaliados a cada } 4 \\
\text { semanas. } 3 x / \text { sem, por } 16 \text { sem, com duração de } 60^{\prime} \text {. Follow-up: } 28 \text { sem. } \\
\text { RESULTADO: } 8 \text { sem = diminuição da dor em todos os grupos. Mais } \\
\text { significativa no G2 (p<0,01). }\end{array}$ \\
\hline $\begin{array}{l}\text { LARSSON } \\
\text { ET AL. } \\
\text { (2015) }\end{array}$ & $\begin{array}{c}\mathrm{n}=130 \\
\mathrm{G} 1: \mathrm{n}=67 \\
\mathrm{G} 4: \mathrm{n}=63\end{array}$ & $\begin{array}{l}\text { G1: Leg-pres, rosca direta, aperto da mão, salto e controle do core. Iniciou } \\
\text { com } 40 \% \text { de } 1 \text { RM (15-10 rep.), na 3-4 sem: } 60 \% \text { 1RM (10-12 rep.), 6-8 } \\
\text { sem: } 80 \% \text { 1RM (5-8 rep.) sendo 1-2 séries, com duração de } 60 \text { '. G4: } \\
\text { Relaxamento autógeno, pensando em partes do corpo individuais } \\
\text { (mentalmente) durante } 25 \text { '. 2x/sem, por } 15 \text { sem. } \\
\text { RESULTADO: Redução da intensidade da dor no grupo G1 (p=0,033). }\end{array}$ \\
\hline $\begin{array}{l}\text { FIGUEROA } \\
\text { ET AL. } \\
(2008)\end{array}$ & $\begin{array}{c}\mathrm{n}=19 \\
\text { G1: } \mathrm{n}=10 \\
\text { G4: } \mathrm{n}=9\end{array}$ & $\begin{array}{l}\text { G1: ERs, de } 8-12 \text { rep. Na máquina para: peitoral, extensores e flexores do } \\
\text { joelho, bíceps e tríceps branquial, reto abdominal. Iniciando com uma } \\
\text { carga de } 50 \% \text { de } 1 \mathrm{RM} \text {, progredindo até as } 16 \text { sem para } 80 \% \text {. G4: sem } \\
\text { intervenção. } 2 x / \mathrm{sem} \text {, por } 16 \text { sem. Duração: } 30 \text { '. }\end{array}$ \\
\hline
\end{tabular}

RESULTADO: Apresentou uma redução da dor de 39\% nas mulheres do $\mathrm{G} 1(\mathrm{p}<0,05)$.

Legenda: G1 = exercício resistido; G2 = exercício aeróbio; G3 = alongamento; N= quantidade de participantes; G4 = controle; SEM= semana; ER = exercício resistido; $\mathrm{RM}=$ repetição máxima; $\mathrm{REP}=$ repetição; $\mathrm{F}=$ flexibilidade.

Oliveira et al. (2017), trabalharam da seguinte forma: 1- adaptação nos três primeiros meses (incluindo o treinamento aeróbico, de força e flexibilidade), 2- transição 
de 4 a 6 meses (inclusão da participação no grupo de apoio psicológico supervisionado), 3- convivência do 7 mês em diante (os participantes se dedicaram somente aos exercícios físicos). O estudo apresenta evidentemente uma melhora significativa do momento inicial $(\mathrm{p}<0,05)$ para o momento final $(\Delta=22,77 \%)$, através dos exercícios de força e flexibilidade. Quando os autores falam de 1- adaptação nos 2 primeiros meses a flexibilidade foi de 24,95 cm; 2- transição de 3-4 meses 28,85 cm e no 5-6 meses 30,63 $\mathrm{cm}$. Nota-se que a diferença entre a pré-intervenção, para os 3 meses foi de $-3,90 \mathrm{~cm}$, da pré-intervenção para os 6 meses $-5,68 \mathrm{~cm}$, e entre os 3 meses e os 6 meses $-1,78 \mathrm{~cm}$. Contudo, essa intervenção de 3 meses de exercício físico, não provocou alterações significativas na flexibilidade. Contrariamente, somente após 6 meses de ingresso que essas pacientes SFM, tiveram resultados/ganhos significativos/positivos quanto a maior facilidade em realizar movimentos de flexibilidade.

Enfatizando a Tabela 1, os estudos de Bircan et al. (2008), Gavi et al. (2014) e Kayo et al. (2012), a carga dos exercícios resistidos foram incrementadas de acordo com a tolerância do paciente, não atingindo assim altas intensidades inicialmente, porém foram observadas diminuição da dor nos pacientes conforme a capacidade progressiva do aumento da carga foram evoluindo através dos treinos contínuos e supervisionados. Diferentemente dos estudos de Larsson et al. (2015) e Figueroa et al. (2008), que variaram a intensidade entre $40 \%$ e $80 \%$ de 1 repetição máxima (RM), iniciando com cargas entre $40-50 \%$ respectivamente e foram progredindo para 70-80\% no final do treinamento. Contudo, independente do parâmetro de intensidade adotado, a resposta quando comparado ao grupo de controle, os autores observaram diminuição das dores no grupo que adotou o treinamento resistido.

\section{Considerações finais}

O treinamento resistido (força) e flexibilidade quando realizado de maneira orientada e monitorado por um profissional de educação física, apresentam resultados eficiente na redução do quadro álgico em pacientes femininos com fibromialgia. Para também minimizar os casos graves dessa doença crônica, deve-se ainda somar com outras intervenções: apoio nutricional e psicológico, terapias complementares como massagem, pilates e o uso de relaxante muscular, para reversão rápida dessa patologia álgica. 


\section{REFERÊNCIAS}

AHLES, T. A.; YUNUS, M. E.; MASI, A. T. A dor crônica é uma variante da doença depressiva? O caso da síndrome de fibromialgia primária. 1987.

AMERICAN COLLEGE OF RHEUMATOLOGY. Empowering Rheumatology Professionals. Disponível em - <https://www.rheumatology.org/I-Am-A/PatientCaregiver/Enfermedades-y-Condiciones/Fibromialgia>. Acesso: 03 de outubro de 2021.

BIRCAN, C.; KARASEL, S. A.; AKGUN, B. E. 1. O.; ALPER, S. Effects of muscle strengthening versus aerobic exercise program in fibromyalgia. Rheumatol Int. 2008.

BILODEAUL K. Harvard Women's Health Watch. Editora executiva, 2020. Disponível em: <https://www.health.harvard.edu/blog/getting-the-best-treatment-foryour-fibromyalgia-2020091020905>. Acesso em: 16 de out. de 2021.

BORCHERS, A. T.; GERSHWIN, M. E. Fibromialgia: uma revisão crítica e abrangente. Clin Rev Allergy Immunol. p. 100-51. 2015.

BULHÕES L. C. C.; LIMA, F. B. F.; FONTES, F. P.; VARELlA, L. R. D.; BRASILEIRO, J. S. Efeito do treinamento resistido na redução da dor no tratamento de mulheres com fibromialgia: revisão sistemática. Rev. Bras. Ci. e Mov. 2018.

COELHO, C. W.; ARAÚJO, C. S. Relação entre aumento da flexibilidade e facilitações na execução de ações cotidianas em adultos participantes de programa de exercícios supervisionado. Rev. Bras. Cineantropom. Desempenho Hum. 2000.

CORRALES, B. S.; LORA, M. H. EI entrenamiento de la fuerza muscular for el tratamiento del síndrome de fibromialgia. Fisioterapia. p. 44-53. 2007.

FIGUEROA, A.; KINGSLEY, J. D.; MCMILLAN, V.; PANTON, L. B. Resistance exercise training improves heart rate variability in women with fibromyalgia. Clin. Physiol Funct Imaging. 2008.

GAVI, M. B. R. O.; VASSALO, D. V.; AMARAL, F. T.; MACEDO, D. C. F.; GAVA, P. L.; DANTAS, E. M. Et. al. Strengthening Exercises Improve Symptoms and Quality of Life but Do Not Change Autonomic Modulation in Fibromyalgia: A Randomized Clinical Trial. PLoS One. 2014.

GRANGES, G.; LITTLEJOHN, G. Limite de dor por pressão em indivíduos sem dor, em pacientes com síndromes de dor regional crônica em pacientes com fibromialgia syndrol. Arthritis Rheum. 1993.

JONES, K. D.; CLARK, S. R.; BENNETT, R. M. Prescribing exercise for people with fibrolmyalgia. AACN. Clin. Issues. 2002.

JUNIOR, M. F.; GOLDENFUM, M. A.; SIENA, C. A. F.; Fibromialgia: clínica e ocupacional. Fibromialgia: aspectos clínicos e ocupacionais. 2012. 
HAUN, M. V. A.; FERRAZ, M. B.; POLLAK, D. F. Validação dos critérios do Colégio Americano de Reumatologia (1990) para classificação da fibromialgia em uma população brasileira. Ver. Bras. Reumatol. 1999.

KAYO, A. H.; PECCIN, M. S.; SANCHES, C. M.; TREVISANI, V. F. M. Effectiveness of physical activity in reducing pain in patients with Fibromyalgia: a blinded randomized clinical trial. Rheumatol Int. 2012.

LARSSON, A.; PALSTAM, A. LÖFGREN, M.; ERNBERG, M.; BJERSING, J.; BILEVICIUTE-LJUNGAR, I. Et. al. Resistance exercise improves muscle strength, health status and pain intensity in fibromyalgia - a randomized controlled trial. available from: <http://dx.doi.org/10.1186/s13075-015-0679-1>. Access: Octub. 20, 2021. Arthritis Research \& Therapy. 2015.

OLIVEIRA, L. H. S.; MATTOS, R. S.; CASTROS J. B. P.; Et. al. Efeito do exercício físico supervisionado sobre a flexibilidade de pacientes com fibromialgia. Rev. Dor. São Paulo, 2017.

PROVENZA, J. R.; POLLAK, D. F.; MARTINEZ, J. E.; PAIVA, E. S.; HELFENSTEIN, M.; HEYMANN, R.; MATOS, J. M. C.; SOUZA, E. J. R. Fibromialgia. 2004.

RUSSELL. Fibrosite / Fibromialgia. In: HYDE, B. M.; GOLDESTEIN, J.; LEVINE, P. Editores. A base clínica e científica da encefalomielite miálgica / síndrome da fadiga crônica. Ottawa: Nightingale Research Foundation. 1992.

SILVA, F. G.; SUDA, E. Y.; MARÇULO, C. A.; PAES, F. H. S.; PINHEIRO, G. T. Comparison of transcutaneous electrical nerve stimulation and hydrotherapy effects on pain, flexibility and quality of life in patients with fibromyalgia. 2008.

SOUZA, J. B.; PERISSINOTTI, D. M. N. A prevalência da fibromialgia no Brasil estudo de base populacional com dados secundários da pesquisa de prevalência de dor crônica brasileira. São Paulo, 2018.

TANGERINO, A. L. A fibromialgia e o acidente de trabalho. Disponível em: $<$ https://jus.com.br/artigos/30244/a-fibromialgia-e-o-acidente-de-trabalho. Acesso em 02 de outubro de 2021.

WOLFE, F.; CLAUW, D. J.; FITZCHARLES, M. A.; GOLDENBERG, D. L.; KATZ, R. S.; MEASE, P. Et. Al. Critérios diagnósticos preliminares do American College of Rheumatology para fibromialgia e medição da gravidade dos sintomas. Arthritis Care Res. p. 600-10. 2010

Recebido em: 01/12/2021

Aprovado em: 23/12/2021

Publicado em: 11/01/2022 